\title{
Parkinsonism and dysautonomia with anti- CV2/CRMP5 associated paraneoplastic neurological syndromes mimicking multiple system atrophy: a case report
}

\author{
Jia Song ${ }^{\dagger}$, Ying Zhang ${ }^{\dagger}$, Yue Lang, Yi-Heng Wang, Jie Shao and Li Cui ${ }^{*}$ (]
}

\begin{abstract}
Background: Paraneoplastic neurological syndromes (PNSs) are broad-spectrum disorders that can affect any part of the nervous system varying in core symptoms. Onconeural antibodies, including Hu, Yo, Ri, anti-CV2, amphiphysin, $\mathrm{Ma2}$, and $\mathrm{Tr}$ are well-characterized and commonly used for the diagnosis of definite PNS. Generally, anti-CV2 antibodies have usually been associated with cerebellar ataxia, chorea, peripheral and autonomic neuropathies, myelopathy, optic neuritis, and retinitis. However, Parkinsonism has not been reported as the core symptom in patients with antiCV2 antibodies.
\end{abstract}

Case presentation: We report a patient with anti-CV2 antibody manifested as Parkinsonism and autonomic dysfunction, which may lead to the diagnosis of multiple system atrophy with predominant Parkinsonism (MSA-P). A lumbar puncture examination was undergone to find a positive anti-CV2 antibody in cerebrospinal fluid. PET-CT showed no tumor. Immunotherapy was adopted and the symptoms were relieved for 5 months. However, with no evidence of tumor, he died after 8 months.

Conclusions: Our findings indicate that PNS with anti-CV2 antibody can be shown as MSA-P mimic. Considering that MSA is a neurodegenerative disease with a poor prognosis, screening for other treatable or controllable factors like PNS presented in this case is necessary when encountering a rapid progressive MSA-mimic patient.

Keywords: Paraneoplastic syndrome, Anti-CV2 antibody, Multiple system atrophy, Collapsin response mediated protein 5, Dysautonomia

\section{Background}

PNS represents the remote effects of tumors on the nervous system, which is not associated with invasion or compression of the tumor but by activation of the immune system. Antibodies produced by the body against the tumor antigen may damage the nervous

\footnotetext{
*Correspondence: Icui@jlu.edu.cn

${ }^{\dagger}$ Jia Song and Ying Zhang contributed equally to this work and share first authorship.

Department of Neurology, Jilin University First Hospital, Xinmin Street 1, Changchun, Jilin, China
}

system. These antibodies are divided into 1) antibodies against neuronal nuclear and cytoplasmic antigens and 2) antibodies against cell surface and synaptic proteins. As a member of the former first identified as a biomarker of PNS in 2001 [1], collapsin response mediated protein 5 (CRMP5) is a neuronal cytoplasmic protein recognized by anti-CV2 antibody expressed in the cerebellum, hippocampus, thalamus, peripheral neurons, spinal cord, retina, and in SCLCs $[1,2]$. Accordingly, the clinical presentations associated with anti-CV2 antibody include cerebellar ataxia, chorea, peripheral and autonomic 
neuropathies, myelopathy, optic neuritis, and retinitis [1, $3,4]$. Anti-CV2 antibody related PNS is closely related to small cell lung cancer and thymoma $[1,3]$. The symptoms can be significantly alleviated after tumor-targeted treatment. Here, we report a case with anti-CV2 associated PNS mimicking MSA without tumor. It reminds physicians of the possibilities for diseases that present as MSA, especially tumor related PNS.

\section{Case presentation}

A 70-year-old man presented to hospital with a 6-month history of slow movements with leaning forward and postural instability. Erectile dysfunction also occurred for more than half a year, before the motor symptoms. A month ago, the symptoms worsened. Meanwhile, he suffered from severe dizziness while standing, constipation and urinary incontinence in this month.. He was a heavy smoker previously without subjective loss of smell, seizures, or changes in body weight. He had no diabetes, family history of genetic diseases or exposure to toxic substances.

His supine blood pressure (BP) was $106 / 76 \mathrm{mmHg}$ with heart rate (HR) 82 beats/ min, while it could not be measured accurately when standing. He showed hypophonia and reduced facial expression. The muscle strength score in extremities was $5 / 5$. He also showed bradykinesia, bilateral reduced arm swing and right upper limbs and both lower limbs rigidity without tremor. The deep tendon reflexes were normal and plantar reflex was flexor bilaterally.

The values of thyroid function test, tumor markers were within the normal range. Results of serum anti-HIV antibody and hepatitis $\mathrm{C}$ antibody were negative. Urinary ultrasound showed $130 \mathrm{~mL}$ of post-void residual urine. Midbrain sonography and thymus computed tomography (CT) were normal. Multi-detector row CT scan of the lungs showed nodules with no thickening of bronchial wall, enlargement of hilar and mediastinal lymph nodes or atelectasis. Brain magnetic resonance imaging (MRI) showed no brain stem or cerebellar atrophy (Fig. 1A). The $11 \mathrm{C}-\mathrm{CFTPET} / \mathrm{CT}$ scan showed reduced uptake of $11 \mathrm{C}$ CFT in the left caudate nucleus and bilateral putamen (Fig. 1B), and 18F-FDGPET/CT scan showed symmetrical hypometabolism of bilateral frontal-parietal lobes (Fig. 1C).

Based on his clinical information, he developed dysautonomia accompanied by Parkinsonism, which led to the diagnosis of MSA-P. According to the second consensus statement on the diagnosis of multiple system atrophy, he met the criteria of probable MSA [5]. Considering his hypotension, levodopa-benserazide $12.5 \mathrm{mg} / 3.125 \mathrm{mg}$ were given orally three times a day. A week later, the dose was doubled. However, he showed no improvement in bradykinesia and instability posture, and the stiffness
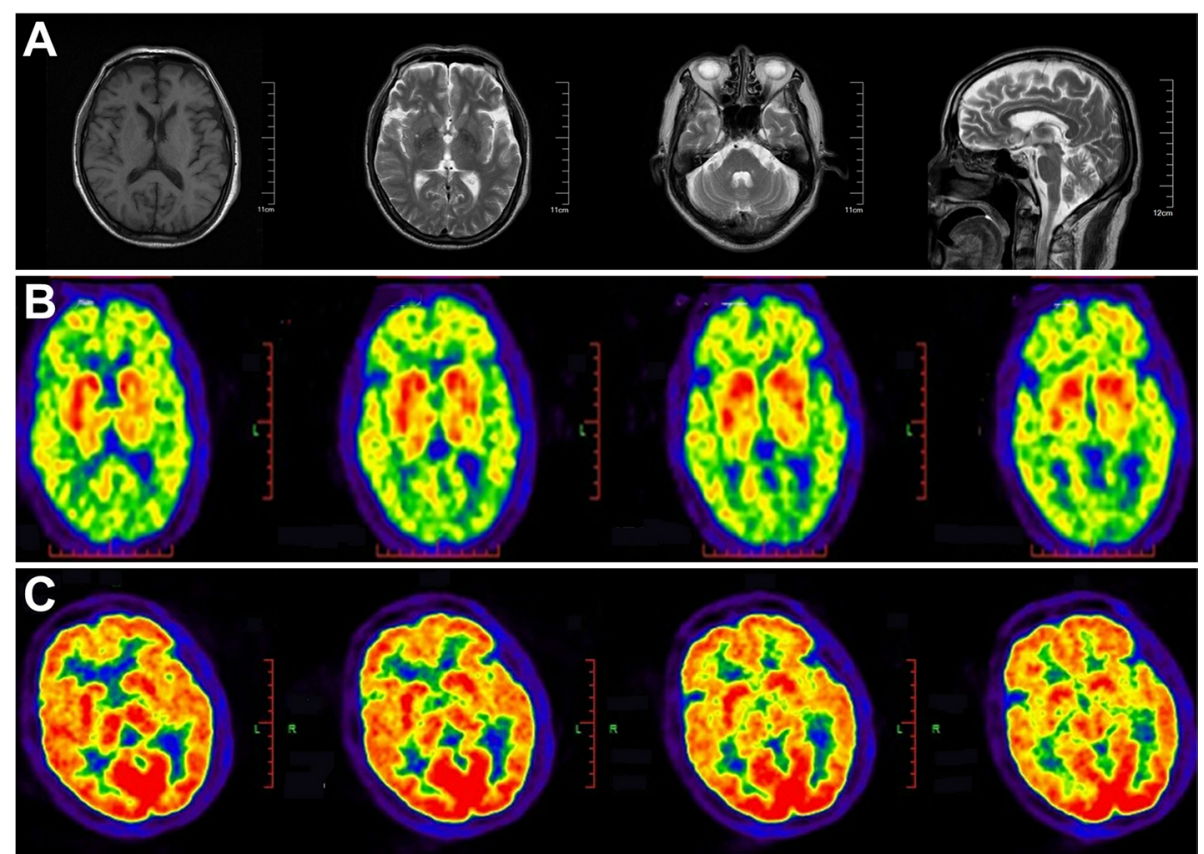

Fig. 1 A Images of the patient's MRI. Brain MRI showed multiple ischemic foci without brain stem or cerebellar atrophy. B Images of the patient's ${ }^{11} \mathrm{C}$-CFTPET/CT. ${ }^{11} \mathrm{C}$-CFTPET/CT scan showed low metabolism of the left caudate nucleus and bilateral putamen. $\mathbf{C}$ Images of the patient's ${ }^{18}$ F-FDGPET/CT. ${ }^{18} \mathrm{~F}$-FDGPET/CT scan showed symmetrical low metabolism of bilateral frontal-parietal lobes 
in the limbs prevented him from moving around at will. What's worse, he suffered from a severe drop in BP, which set him free from a levodopa trial. Meanwhile, he was recommended to wear elastic socks, raise head $30^{\circ}$ when lying, use abdominal straps when standing and drink adequate water. At the same time, droxidopa $100 \mathrm{mg}$ was given orally once a day. However, his BP did not improve significantly. In case of supine hypertension, the dose was not increased and midodrine was not used.

Meanwhile, his dysautonomia progressed relatively rapidly. There was no supportive imaging evidence for MSA. Considering that MSA is a neurodegenerative disorder with no effective treatment and poor prognosis, we checked for other treatable or controllable factors. $\mathrm{He}$ underwent a lumbar puncture examination. In the cerebrospinal fluid (CSF), protein was $460 \mathrm{mg} / \mathrm{L}$ and total leukocyte count was $4.00 \times 10^{6} / \mathrm{L}$. The anti-CV2 antibody was positive in the CSF and negative in the serum. Then a whole-body PET-CT scan was done and no tumor was detected. Based on the recommended diagnostic criteria for PNS [3], we diagnosed the patient as definite PNS with the nonclassical neurological syndrome, wellcharacterized onconeural antibodies, and no cancer.

Intravenous immunoglobulin was given at $0.4 \mathrm{~g} / \mathrm{kg} /$ day. After 5 days of immunotherapy, his dizziness was significantly improved and he could walk independently. His supine BP was $130 / 83 \mathrm{mmHg}$ with HR 79 beats/ min. When standing, his BP was $123 / 75 \mathrm{mmHg}$ with HR 82 beats/min at $1 \mathrm{~min}$ and $115 / 66 \mathrm{mmHg}$ with HR 85 beats/min at $3 \mathrm{~min}$. The BP was higher than that on admission with the absence of orthostatic hypotension. He received active hydration and medication every day since admission. However, the BP did not improve until the use of immunoglobulin, suggesting that it was the immunotherapy that worked. Moreover, there was no post-void residual urine in reexamination. As the symptoms relieved, intravenous immunoglobulin was stopped after 10 days. In consideration of the age and side effects, immunotherapy was refused by his relatives in the follow-up therapy. Considering that neurological symptoms may appear before the discovery of tumor in some PNS patients, we a followed him up dynamically.

Five months later, his symptoms worsened characterized by frequent falls, inability to walk independently, dysphagia and unintelligible speech. His limbs were too tense to be bent. Likewise, no positive results were found in tumor screening. The BP was $140 / 89 \mathrm{mmHg}$, and levodopa-benserazide $50 \mathrm{mg} / 12.5 \mathrm{mg}$ were given orally three times a day for months. However, it did not work. Unfortunately, he died after 8 months. We also organized the patient's clinical manifestations and treatment options as a timeline (Fig. 2).

\section{Discussion and conclusions}

The patient's clinical presentation was classified as "nonclassical neurological syndrome", indeed, the presentations of dysautonomia and Parkinsonism in anti-CV2 antibody related PNS are rare. Although autonomic neuropathy is taken as common signs, only seven patients showed Parkinsonism without severe and rapidly progressive dysautonomia [1, 6-9]. Here, we summarized the clinical characteristics of these 7 patients in tabular form (Additional Table 1).

Among the 7 patients (excluding this case), the ratio of male: female was approximately equal $(42.9 \%)$. The mean age of onset was 60.3 (range $45-72$ years old) during the 4 patients whose ages were recorded. Clinical manifestations were described in detail in 4 patients,

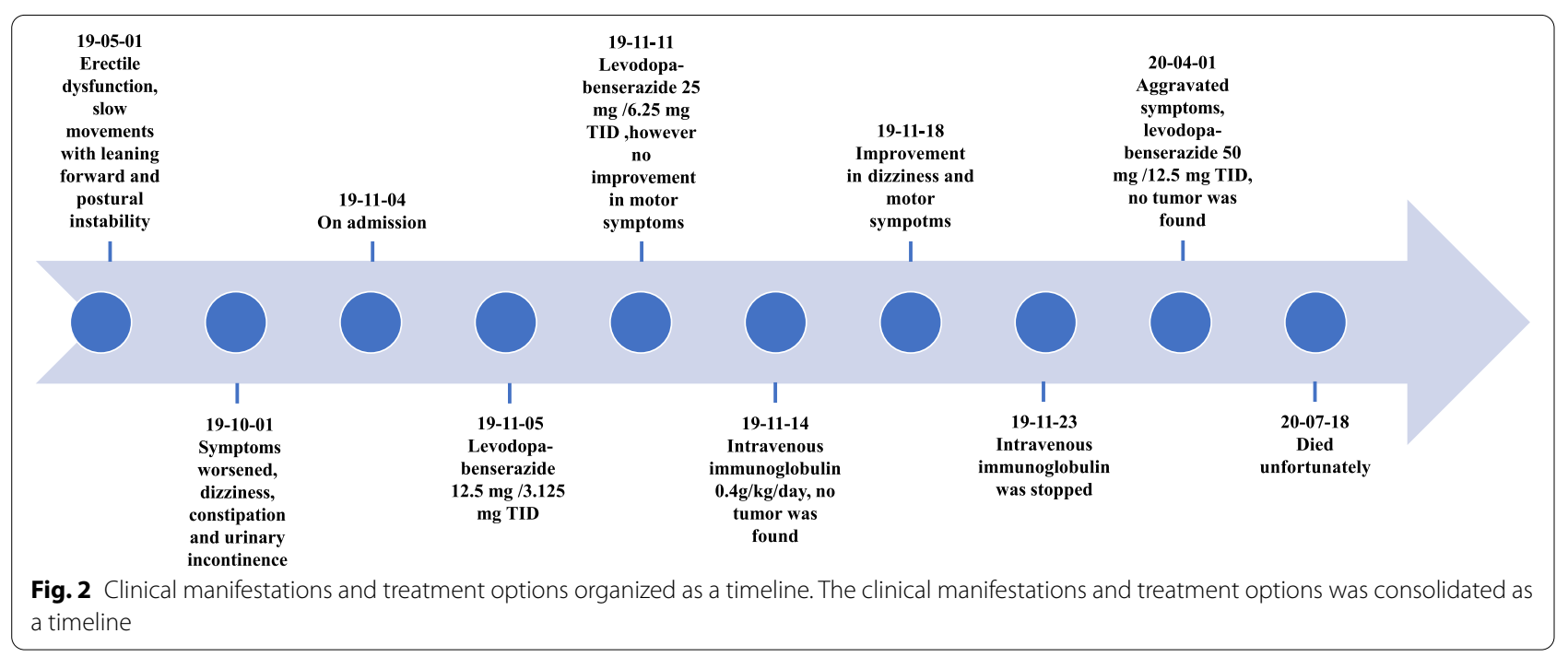


with concomitant symptoms including: dysarthria $(n=1,25 \%)$ hoarse voice $(n=1,25 \%)$, autonomic nerve damage $(n=2,50 \%$, one constipation, the other urinary urgency), sleep disturbance ( $n=1,25 \%$, core symptom), and loss of taste $(n=1,25 \%)$. Basal ganglia changes were found in all the 4 patients (100\%) whose brain MRI results were described, which could account for Parkinsonism. One case was combined with the thalamus and brainstem changes (25\%). Two cases of MRS examination showed a reduction of NAA peak located in bilateral basal ganglia $(n=1,50 \%)$ and bilateral lateral ventricles $(n=1,50 \%)$. Lumbar puncture results were shown in 4 cases, 2 patients had elevated CSF protein levels (50\%), with a maximum CSF protein of $629 \mathrm{mg} / \mathrm{L}$. Three patients underwent EEG examination, and 2 showed abnormal results $(66.7 \%)$ with slow waves in the temporal region. Four cases described the sites of antibody in detection, 2 in both blood and cerebrospinal fluid (50\%), and 2 merely in blood (50\%). Four cases gave the information about complicated tumor, treatment and prognosis. Two patients suffered from SCLC (50\%), and radiotherapy (1 case, 50\%) and chemotherapy (2 cases, $100 \%)$ were adopted to perform clinical improvements. One patient diagnosed with breast cancer (25\%) got improvements in life quality after surgery. No tumor was found in one patient (25\%) who received hormone treatment: dexamethasone $10 \mathrm{mg}$ for 3 days and $15 \mathrm{mg}$ for 30 days by intravenous drip, then $6 \mathrm{mg}$ orally once a day with $0.75 \mathrm{mg}$ reduced every 7 days. During the following up, the patient suffered from originally uncontrollable tremors and new onset of seizure. In all, patients achieved cancer treatment when tumors were found are more related to better outcomes than patients accessed to immunotherapy with no tumor.

Here, we report the first case of anti-CV2 antibodypositive PNS mimicking MSA-P. We speculate antibodies damage CRMP5 distributed in the basal ganglia and autonomic nervous system. However, the mechanism remains unknown. PNS treatments consist of tumortargeted therapy, immunotherapy, and symptomatic treatment. Immunotherapy is indicated in patients with positive anti-CV2 antibody acting as pain, gross or fine motor skills, impairment in speech function. However, in general, immunotherapy is not effective in PNS patients with intracellular antibodies such as anti-CV2 antibody [4]. Instead, the symptoms can be significantly alleviated after targeted treatment. The prognosis of PNS has a great relationship with the type of primary tumor and onconeural antibody. Dubey et al. [4] found that patients with CRMP5 have a better 5-year survival rate than patients with ANNA1.

In addition to PNS, many diseases exhibit Parkinsonism and dysautonomia simultaneously (Additional
Table 2). Physicians should make a comprehensive differential diagnosis as follows especially treatable diseases: 1) Neurodegenerative diseases: MSA-P, Parkinson's Disease, Dementia with Lewy bodies. They are mostly shown as slow onset with old age and rare family history. Parkinsonism and autonomic dysautonomia can occur with different core symptoms. Neuroimaging shows characteristic features. There is no effective treatment with poor prognosis, so it is necessary to exclude other treatable diseases. 2) Hereditary degeneration diseases: fragile X-associated tremor ataxia syndrome (FXTAS), spinocerebellar ataxia (SCA), Perry syndrome. SCA and Perry syndrome are autosomal dominant, while FXTAS $\mathrm{X}$ chromosome dominant. The patients suffering from hereditary degeneration diseases are younger than those of neurodegenerative diseases with slow progression. Cerebellar ataxia often acts as the core symptom and some patients may display Parkinsonism and autonomic dysfunction. Genetic testing is reliable. Likewise, there is no effective treatment requiring to be carefully identified. 3) Hereditary metabolic diseases: Wilson's disease, Gaucher's disease-related to Parkinsonism. They are genetically related disorders at a young age and slow onset of abnormal cellular metabolism inherited as an autosomal recessive pattern. Wilson's disease is a copper accumulation disorder, while Gaucher's disease is a lysosomal metabolism disorder. Multiple organs are involved, and the nervous system tends to be one part. Genetic or laboratory testing can help with the confirmation of diagnosis. As for treatment, dietary changes and replacement therapy with enzymes have a positive effect on patients. We need to take this kind of disease into consideration as the treatment can alleviate the symptoms. 4) Infection and autoimmune diseases: Sjogren's syndrome, acquired immune deficiency syndrome [10]. Patients have histories of infection or autoimmune diseases. Symptoms can deteriorate rapidly. Etiological examination or pathological biopsy is probative for diagnosis. It has to be emphasized that immunotherapy leads to a better prognosis, thereby the possibility of such diseases should be considered preferentially. 5) PNS [11]. Older patients showed rapid progression and risk factors associated with tumors. Generally, clinical manifestations are closely related to the type of antibody. Examination of paraneoplastic antibodies in the blood and CSF and the screening for tumors is required. Treatment aiming at tumors can relieve symptoms. Even with no tumor found, immunotherapy can relieve symptoms for some time as is described in this report. Therefore, we should actively detect PNS-related antibodies and the presence of primary tumors in patients with MSA symptoms. 6) Systemic light chain amyloidosis [12]. It's a rare disease caused by extracellular deposition of amyloid 
with rapid progression. Symptoms are related to organs where amyloid is deposited (such as the heart, liver, kidney, and peripheral nerves) with a case report showing MSA-mimic. Tissue biopsy or abdominal liposuction is convincing. Patients are commonly treated with chemotherapy, but the prognosis is generally poor for organ complications. Given that, it is of great significance to check for treatable factors.

In this study, we report a case of anti-CV-2-associated Parkinsonism and rapidly progressive dysautonomia. The characteristics are as follows: 1 ) PNS patients with anti$\mathrm{CV}-2$ antibody have been reported to be associated with Parkinsonism (Additional Table 1), yet no dysautonomia or mild dysautonomia were described in these cases. Here, we reported a patient associated with anti-CV-2 antibody with rapid progression (compared with MSA-P patients) of severe autonomic nervous symptom impairment and Parkinsonism, which is difficult to differentiate from MSA-P in clinical manifestations. We speculate that CRMP5 is expressed in both autonomic nervous system and basal ganglia, as evidenced by reduced uptake of $11 \mathrm{C}$-CFT in the left caudate nucleus. 2) Tumor-specific treatment is beneficial to clinical outcomes in patients with PNS. However, our patient was repeatedly screened for tumors to show negative results, and immunotherapy is a valuable treatment for temporary relief of symptoms. Therefore, on the one hand, screening for tumors should be carried out throughout the course of the disease, on the other hand, immunotherapy can be taken as an alternative as early as possible when no tumor is found.

However, there are limitations in this case: 1) Considering the absence of evidence of tumors and economic reasons, the patient did not recheck PET-CT and the antibody after immunotherapy and during his follow-up. 2) Autopsy was not performed after death, so the exact cause of death could not be determined, nor could it be determined whether the patients had antigen-antibody complex deposition and pathological changes in basal ganglia and autonomic nervous system in the brain tissue, so as to explore the mechanism of anti-CV-2 antibody in cerebrospinal fluid causing Parkinsonism and autonomic nervous dysfunction. 3) Due to limited conditions, the titer of anti-CV-2 antibody in cerebrospinal fluid and the specific binding ratio of dopamine were unknown. This study reminds physicians of the differential diagnosis of MSA-P, especially PNS when encountering patients with Parkinsonism and rapidly progressing autonomic failure.

\section{Abbreviations}

AIDS: Acquired immune deficiency syndrome; BP: Blood pressure; CRMP5: Collapsin response mediated protein 5; CSF: Cerebrospinal fluid; CT: Computed tomography; DLB: Dementia with Lewy bodies; ED: Erectile dysfunction; EEG:
Electroencephalography; FDG PET: Fluorodeoxyglucose positron emission tomography; FLAIR: Fluid-attenuated inversion recovery; FXTAS: Fragile X-associated tremor ataxia syndrome; HIV: Human immunodeficiency virus; HR: Heart rate; MIBG: Meta-iodobenzylguanidine; MRI: Magnetic resonance imaging; MRS: Magnetic resonance spectroscopy; MSA-P: Multiple system atrophy predominant Parkinsonism; NAA: N-acetylaspartate; OH: Orthostatic hypotension; PD: Parkinson's disease; PET: Positron emission tomography; PNS: Paraneoplastic neurological syndromes; REM: Rapid eye movement; SCA: Spinocerebellar ataxia; SCLC: Small-cell lung carcinoma.

\section{Supplementary Information}

The online version contains supplementary material available at https://doi. org/10.1186/s12883-021-02448-6.

Additional file 1: Table 1. Clinical characteristics of anti-CV-2 related PNS presenting with Parkinsonism.

Additional file 2: Table 2. Summary of diseases manifested as Parkinsonism and dysautonomia.

\section{Acknowledgments}

The authors acknowledge the contribution of the patient and his agreement to have his case published.

\section{Authors' contributions}

JSo, YZ: Study concept and design, acquisition of data, drafting the article. YL, Y-HW, JSh: acquisition of data, analysis and interpretation. LC: critical revision of the manuscript for important intellectual content. All authors have read and approved the manuscript.

Funding

This research did not receive any specific grant from funding agencies in the public, commercial, or not-for-profit sectors.

Availability of data and materials

Data sharing is not applicable to this article as no datasets were generated or analyzed during the current study.

\section{Declarations}

Ethics approval and consent to participate

Not applicable.

\section{Consent for publication}

Written informed consent was obtained from the patient's next of kin for publication of this case report and any accompanying images. A copy of the written consent is available for review by the Editor-in-Chief of this journal.

\section{Competing interests}

The authors declare that they have no competing interests.

Received: 24 May 2021 Accepted: 18 October 2021

Published online: 26 October 2021

\section{References}

1. Yu Z, Kryzer TJ, Griesmann GE, Kim KK, Benarroch EE, Lennon VA. CRMP-5 neuronal autoantibody: Marker of lung cancer and thymoma-related autoimmunity. Ann Neurol. 2001;49:146-54.

2. Ricard D, Rogemond V, Charrier E, Aguera M, Bagnard D, Belin MF, et al. Isolation and expression pattern of human Unc-33-like phosphoprotein 6/collapsin response mediator protein 5 (Ulip6/CRMP5): Coexistence with Ulip2/CRMP2 in Sema3A-sensitive oligodendrocytes. J Neurosci. 2001;21:7203-14. 
3. Graus F, Delattre JY, Antoine JC, Dalmau J, Giometto B, Grisold W, et al. Recommended diagnostic criteria for paraneoplastic neurological syndromes. J Neurol Neurosurg Psychiatry. 2004;75:1135-40.

4. Dubey D, Lennon VA, Gadoth A, Pittock SJ, Flanagan EP, Schmeling JE, et al. Autoimmune CRMP5 neuropathy phenotype and outcome defined from 105 cases. Neurology. 2018;90:e103-10.

5. Gilman S, Wenning GK, Low PA, Brooks DJ, Mathias CJ, Trojanowski JQ, et al. Second consensus statement on the diagnosis of multiple system atrophy. Neurology. 2008;71:670-6.

6. Tada S, Furuta M, Fukada K, Hirozawa D, Matsui M, Aoike F, et al. Severe parkinsonism associated with anti-CRMP5 antibody-positive paraneoplastic neurological syndrome and abnormal signal intensity in the bilateral basal ganglia. J Neurol Neurosurg Psychiatry. 2016;87:907-10

7. Yap SM, Lynch T, MacMahon P, Murray B. Paraneoplastic Atypical Parkinsonism with Anti-CRMP5 Antibodies and Severe Caudate and Putaminal Hypometabolism on 18-Fluorodeoxyglucose Positron Emission Tomography of the Brain. Mov Disord Clin Pract. 2017;4:263-5.

8. Cheng Y, Liu K, Liu S, Meng H, Zheng X, Zhang Y, et al. Anti-CV2 autoimmune encephalitis: a case report and review of the literatures. Chin J Neurol. 2018;51(5):376-81. https://doi.org/10.3760/cma.j.issn.1006-7876. 2018.05.009.
9. Wu X, Wang H, Xu G, Lin Y. Anti-CV2 Autoimmune Encephalitis With Parkinson-Like Symptoms and Bilateral Leukoencephalopathy-A Case Report. Front Neurol. 2019;10(October):1-5.

10. Kurihara M, Sasaki T, Ishiura H, Tsuji S. HIV dementia with a decreased cardiac 123 i-metaiodobenzylguanidine uptake masquerading as dementia with lewy bodies. Intern Med. 2018;57:3007-10.

11. Ricigliano VAG, Fossati B, Saraceno L, Cavalli M, Bazzigaluppi E, Meola G MSA mimic? Rare occurrence of anti-hu autonomic failure and thymoma in a patient with parkinsonism: Case report and literature review. Front Neurosci. 2018;12(JAN):1-6.

12. Albanese A, Cocco A, Milani P, Lalli S, Palladini G. Parkinsonism and dysautonomia: Multiple system atrophy? Parkinsonism Relat Disord. 2020;77:146-9.

\section{Publisher's Note}

Springer Nature remains neutral with regard to jurisdictional claims in published maps and institutional affiliations.
Ready to submit your research? Choose BMC and benefit from:

- fast, convenient online submission

- thorough peer review by experienced researchers in your field

- rapid publication on acceptance

- support for research data, including large and complex data types

- gold Open Access which fosters wider collaboration and increased citations

- maximum visibility for your research: over $100 \mathrm{M}$ website views per year

At BMC, research is always in progress.

Learn more biomedcentral.com/submissions 